Research Paper

\title{
Benefits of resistant starch type 2 for patients with end-stage renal disease under maintenance hemodialysis: a systematic review and meta-analysis
}

\author{
Linpei Jia, MD, $\mathrm{PhD}^{1}$, Xingtong Dong, MD${ }^{1}$, Xiaoxia Li, MD ${ }^{1}$, Rufu Jia, MD ${ }^{\circledR}$ and Hong-Liang Zhang, \\ $\mathrm{MD}, \mathrm{PhD}^{3}$ \\ 1. Department of Nephrology, Xuanwu Hospital, Capital Medical University, Changchun Street 45\#, 100053, Beijing, China. \\ 2. Central Hospital of Cangzhou, Xinhua Middle Street 201\#, 061001, Cangzhou, Hebei Province, China. \\ 3. Department of Life Sciences, National Natural Science Foundation of China, Shuangqing Road 83\#, 100085, Beijing, China. \\ $\triangle$ Corresponding authors: Dr. Linpei Jia, Department of Nephrology, Xuanwu Hospital, Capital Medical University, Changchun Street 45\#, 100053, Beijing, \\ China, E-mail: anny_069@163.com; Dr. Rufu Jia, Central Hospital of Cangzhou, Xinhua Middle Street 201\#, 061001, Cangzhou, China, E-mail: \\ zxyy5688@126.com; and Dr. Hong-Liang Zhang, Department of Life Sciences, National Natural Science Foundation of China, Shuangqing Road 83\#, 100085, \\ Beijing, China, Email: drzhl@hotmail.com.
}

(1) The author(s). This is an open access article distributed under the terms of the Creative Commons Attribution License (https://creativecommons.org/licenses/by/4.0/). See http://ivyspring.com/terms for full terms and conditions.

Received: 2020.08.03; Accepted: 2020.11.23; Published: 2021.01.01

\begin{abstract}
Background: Resistant starch type 2 (RS2) has been documented to regulate gut microbiota and to improve the clinical outcomes of several diseases. However, whether RS2 may benefit patients with end-stage renal disease under maintenance hemodialysis (MHD) remains unknown.

Methods: We conducted a systemic review and meta-analysis of randomized controlled trials (RCTs). Adult patients receiving MHD were treated with RS2 (CRD42020160332). The primary outcomes were changes of uremic toxins, and the secondary outcomes were changes of inflammatory indicators, albumin and phosphorus.

Results: After screening 65 records, five RCTs $(n=179)$ were included. A significant decrease of blood urea nitrogen (weighted mean difference $(\mathrm{WMD})=-6.91,95 \% \mathrm{Cl}:-11.87$ to $-1.95,1^{2}=0 \%, P=0.006$ ), serum creatinine (WMD $=-1.11,95 \% \mathrm{Cl}:-2.18$ to $-0.05,12=44 \%, P=0.04$ ) and interleukin (IL) -6 in blood (standard mean difference (SMD) $=-1.08,95 \% \mathrm{Cl}:-1.64$ to $-0.53,12=35 \%, P=0.0001$ ) was revealed in the RS2 group. Analyses of blood levels of uric acid, p-cresyl sulfate, indoxyl sulfate, high sensitive C-reaction protein, albumin and phosphorus yielded no significant difference.

Conclusions: Our results suggest that RS2 may improve the residual renal function of patients under MHD and mitigate a proinflammatory response.
\end{abstract}

Key words: resistant starch type 2; hemodialysis; renal function; uremic toxin; proinflammatory response

\section{Introduction}

The growing prevalence of chronic kidney disease (CKD) threatens the public health, which leads to a high incidence of end-stage renal disease (ESRD) $[1,2]$. Maintenance hemodialysis (MHD) is a common method of renal replacement therapy for patients with ESRD. Although MHD may improve the life expectancies of patients [3], complications are increasingly recognized [4], such as metabolic syndrome, neurodegeneration and gut dysbiosis [5, 6].
Manipulation of gut microbiota is viewed as a promising approach to deal with a variety of diseases, such as neuropsychiatric disorders, CKD, and so forth [7]. Due to an impaired ability of eliminating uremic toxins, specific uremic milieu of CKD impairs the intestinal barrier and promotes gastrointestinal inflammation, which in turn damages the microbial diversity in CKD patients [8-10]. As kidney is a high-flow organ receiving one quarter of the whole blood volume, with limited anti-inflammatory or 
antioxidant ability [10], gut dysbiosis may aggravate the accumulation of uremic toxins in the body, whereby increasing micro-inflammation in the kidney [11]. As a consequence, the increasingly augmented inflammation further damages renal function and forms a vicious circle.

Prebiotics are typically specialized nondigestible plant fiber compounds that circulate undigested through the upper gastrointestinal tract and enhance the activity of beneficial bacteria in the gut, presenting a favorable effect on the prognosis of CKD [12]. Specific dietary supplements, including resistant starch (RS) [13], are frequently referred to as a modulator of gut flora, yet comprehensive insights into functional responses of microbiota to modulators are largely lacking. As a kind of special starch with high amylose and specific structures of amylopectin molecule [14], RS can escape the digestion in the small intestine and transit to colon, where it can be fermented by specialized members of bacteria [15]. RS type 2 (RS2), in its natural granular form mainly in uncooked potato, corn, and green-banana flours, has been categorized as a prebiotics [16]. In the colon, RS2 serves as a substrate to promote the growth of some beneficial bacteria, like Bacteroidetes, Proteobacteria and so forth [17]. As a fermentable fiber, RS2 increases the production of short chain fatty acids (SCFAs), which reduce the permeability of intestinal walls and further inhibit renal microinflammation [17]. High fiber dietary intake has been associated with a low risk of systemic inflammation and cardiovascular events in patients under MHD [18]. A randomized controlled trial (RCT) in 56 patients under MHD showed that the levels of p-cresyl sulfate (PCS) and indoxyl sulfate (IS) were decreased after administration of RS2 for 6 weeks [19]. Creatinine and inflammation biomarkers, including malondialdehyde, tumor necrosis factor- $\alpha$ and interleukin (IL)-6, were significantly decreased as well after RS2 treatment [20]. Collectively, RS2 appears as a promising adjuvant therapy for patients under MHD. However, conclusions are inconsistent among studies on RS2 in CKD [19-23]. Since no consensus for the clinical use of RS2 has been reached thus far, we herein evaluate the therapeutic effects of RS2 on MHD via an evidence-based method.

\section{Methods}

To investigate the therapeutic effectiveness of RS2 on CKD, we searched for RCTs and analyzed changes of uremic toxins, inflammatory and other clinical indicators in eligible studies through a meta-analysis. Furthermore, we highlighted gaps in literature for guiding clinical studies in this area in the future. We conducted our meta-analysis according to the Preferred Reporting Items for Systematic Reviews and Meta-Analyses (PRISMA) statement (Supplementary file 1). The study has been registered at the International Prospective Register of Systematic Reviews (https://www.crd.york.ac.uk/PROSPERO/, No. CRD42020160332).

\section{Searching strategy}

We combined entry terms of "chronic kidney disease", "end stage renal disease", "resistant starch" and synonyms of these terms to search articles in four major electronic databases, namely PubMed, EMBASE, Web of Science and Cochrane Library (see Supplementary file 2 for the full search strategy). All articles published in the English language as of 20 October 2019 were searched without restrictions of origin of countries or article type. Internet-based information and conferences indexed in four electronic databases were also reviewed. Reference lists of all searched publications were screened to identify missing studies in the initial search by two independent researchers (XD and LJ).

\section{Study selection}

Two researchers (XL and HLZ) assessed the initially yielded publications independently. Firstly, titles and abstracts were screened for appropriate studies. Then full-texts were assessed according to the inclusion criteria. Disagreement on eligibility was addressed by discussion and concluded after consensus. Patients under MHD refer to those with ESRD who had received hemodialysis for at least 3 months [24]. The inclusion criteria were: (1) RCTs regardless of a design of blindness; (2) adult CKD patients receiving regular hemodialysis; (3) RS2 supplemented at any frequency and dosage. If the data of one cohort were published in several articles, the article with the longest therapeutic period and the largest sample size was selected. The exclusion criteria were: (1) non-MHD dependent CKD patients; (2) animal or in vitro experiments; (3) non-RCT clinical studies; (4) editorials, reviews, or publications without full-texts (i.e. conference abstracts); (5) studies using multiple supplements together as one intervention.

\section{Outcome measures}

The major aim of our study was to evaluate the effect of RS2 on renal functions in patients with ESRD under MHD. Fasting blood samples were obtained in the morning before the hemodialysis session. The primary outcomes interdialysis changes of blood urea nitrogen (BUN), serum creatinine (Scr), uric acid (UA), PCS and IS. The secondary outcomes were changes of IL-6, high sensitive C-reaction protein (hsCRP), serum phosphorus and albumin. All 
indicators were measured in serum except that PCS and IS were measured either in serum or plasma.

\section{Data extraction}

Characteristics of studies (researchers, publication year, design of blindness and sample size), demographic data of subjects (country, mean age and ratio of males), details of RS2 treatment (dosage, frequency and duration) and data of outcomes were extracted from each eligible trial by two researchers (XL and HLZ) independently. Discrepancies were judged by a third researcher (RJ) to ensure the accuracy.

\section{Risk of bias and quality assessment}

The risk of bias for each selected study was estimated taking into consideration random sequence generation, allocation concealment, blinding of patients, blinding of outcome assessment, completeness of outcome data, selective reporting and other bias by the Cochrane Collaboration's tool for assessing the risk of bias [25]. Quality of evidence was graded according to the risk of bias, inconsistency, indirectness, imprecision, and publication bias [26] with the Grading of Recommendation Assessment (GRADE) approach by the GRADEpro GDT 2015 (http://gradepro.org). Quality assessment and summary of findings (SoF) were performed by two independent researchers (LJ and XD). Disagreements were resolved with the help of a third researcher (HLZ) if necessary.

\section{Summary measures}

Because all outcome data were continuous variables, data were synthesized by the inverse variance method and the random effects model. Variables were expressed as weighted mean difference (WMD). If variables were in different units or with great differences of measurements among included studies, standard mean difference (SMD) was used.

\section{Statistical analysis}

We used the Review Manager (RevMan 5.3, The Nordic Cochrane Centre, Cochrane Collaboration, Copenhagen, Denmark) to perform the statistical analysis. $P<0.05$ indicated a statistical significance. An inverse variance model was applied for continuous variables. We deployed a random effects model for a better accommodation of heterogeneity. A chi-square test on $\mathrm{n}-1$ degrees of freedom was quantified for statistical heterogeneity [27]. The Cochrane $I^{2}$ statistics were calculated to evaluate the heterogeneity among studies. Heterogeneity of data was considered to be acceptable if the $I^{2}$ value < $50 \%[28]$.

\section{Results}

\section{Study selection and characteristics}

The detailed procedure of literature searching and selection is shown in Figure 1. A total of 65 publications were yielded after the initial searching. After removing 29 duplicates, we excluded an extra of 26 records via screening the titles and abstracts. Further, 5 records, including 2 conference abstracts and 3 reviews $[17,29,30]$, were removed. Finally, 5 RCTs [19-23] including 179 patients under MHD were included after reviewing the full-texts (Figure 1). The summary of the included studies was shown in Table 1. Baseline characteristics of subjects in each study were summarized (Table 2). RS2 from two Ingredion companies (United States and Australia) was used for 4 weeks to 2 months.

\section{Risk of bias and SoF}

We evaluated the risk of bias for each enrolled trial based on the Cochrane Collaboration's tool for assessing the risk of bias (Supplementary file 3). The allocation concealment and the blinding of outcome assessment were assessed as $100 \%$ unclear risk, while the reporting bias was assessed as $100 \%$ low risk (Figure 2a). Esgalhado et al.'s study and Khosroshahi et al.'s study, in 2018 respectively, were evaluated as high risk studies (Figure $\mathbf{2 b}$ ), which might reduce the credibility of our study findings.

We then graded the quality of each outcome and presented the results as an SoF table (Table 3). Among all outcomes, IS and Scr were assessed as very lowquality because of the small sample size, inconsistent study results and the reporting bias. For outcomes assessed as very low-quality, we were uncertain about the estimates. PCS, BUN, UA, IL-6, hsCRP, albumin and phosphorus were graded as low-quality outcomes. For outcomes assessed as low-quality, we were uncertain about the estimates as well, and our estimates might be changed pending further investigations.

\section{Synthesis of results}

Primary outcomes

Scr, BUN and UA are commonly used to evaluate the residual kidney function in the clinical practice. For the RS2 group, levels of BUN (WMD = $-6.91,95 \%$ CI: -11.87 to $-1.95, I^{2}=0 \%, P=0.006$, Figure 3a) and Scr (WMD $=-1.11,95 \%$ CI: -2.18 to $-0.05, I^{2}=$ $44 \%, P=0.04$, Figure $3 \mathbf{b}$ ) were significantly decreased as compared to the control group. The level of UA did not vary between the RS2 group and the control group (WMD $=0.17,95 \%$ CI: -0.23 to $0.58, I^{2}=0 \%, P=$ 0.40 , Figure 3c). 


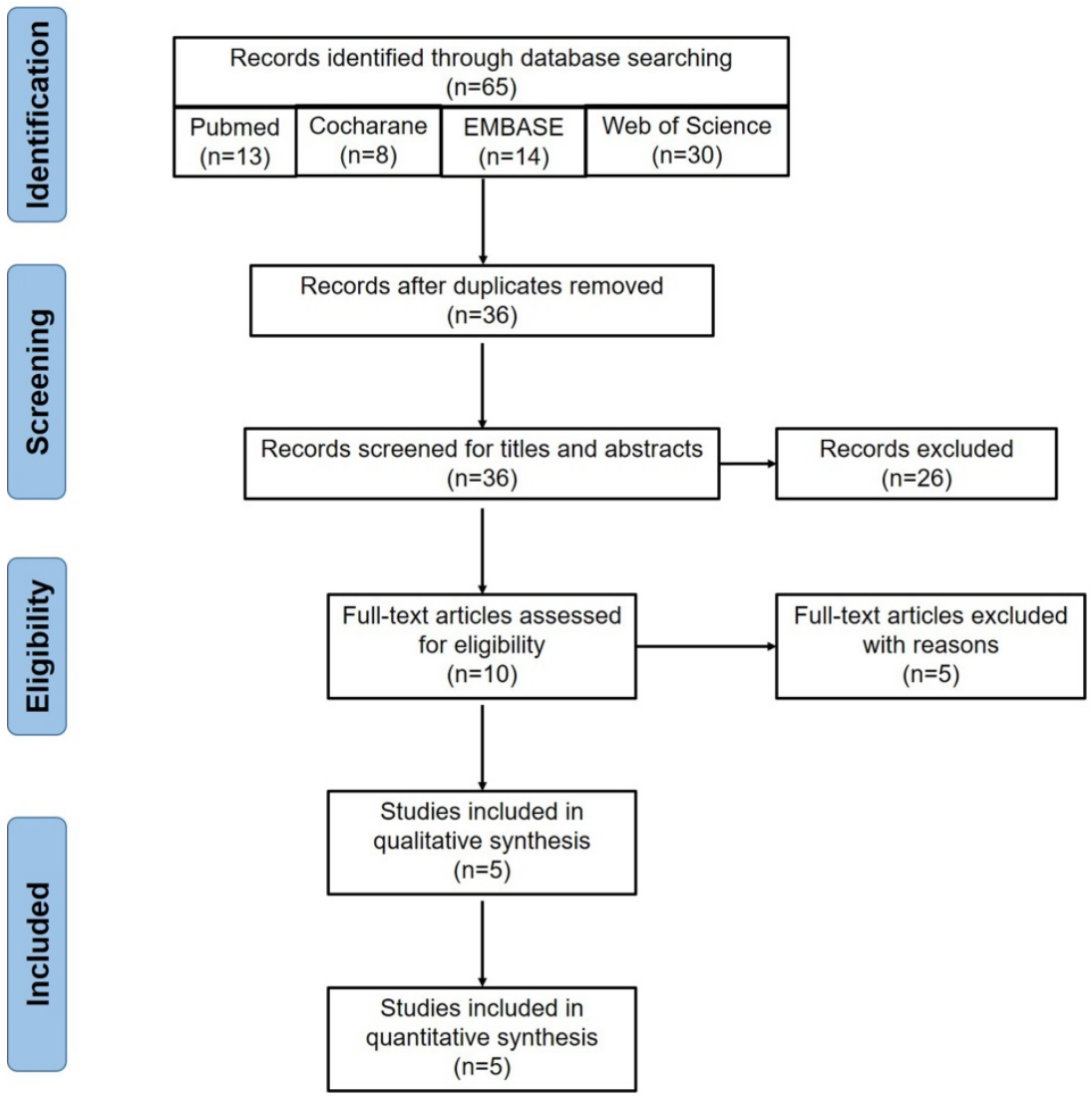

Figure 1. Flow chart of eligible studies screening. Initially, we searched 65 articles from the four databases, including 13 in PubMed, 14 in EMBASE, 30 in Web of Science and 8 in the Cochrane Library. Twenty-nine duplicates were removed. Then 26 publications were excluded after screening titles and abstracts, including 5 animal experiments, 13 non-chronic kidney disease studies, 5 non-resistant starch studies and 3 clinical trial registrations. Five articles were further excluded after reviewing the full-texts, including 3 reviews and 2 conference abstracts. Finally, 5 publications were included for the meta-analysis.

Table 1. The summary of included studies for meta-analysis

\begin{tabular}{|c|c|c|c|c|c|}
\hline Study & Country & Sample size & Formation of resistant starch type 2 (RS2) and placebo & Dosage & $\begin{array}{l}\text { Duration of } \\
\text { treatment }\end{array}$ \\
\hline Sirich 2014 [19] & $\begin{array}{l}\text { United } \\
\text { States }\end{array}$ & $\begin{array}{l}20 \text { patients and } 20 \\
\text { controls }\end{array}$ & $\begin{array}{l}\text { RS2: } 15 \text { g of high-amylose corn starch (Hi-maize } 260) \text {, composed } \\
\text { of approximately } 40 \% \text { digestible starch and } 60 \% \text { RS2; } \\
\text { Placebo: } 15 \text { g of waxy corn starch. }\end{array}$ & $\begin{array}{l}1 \text { sachet daily for } 1 \text { one } \\
\text { week and } 2 \text { sachets daily } \\
\text { for next weeks }\end{array}$ & 6 weeks \\
\hline Esgalhado 2018 [22] & Brazil & $\begin{array}{l}15 \text { patient and } 16 \\
\text { controls }\end{array}$ & $\begin{array}{l}\text { RS2: } 16 \mathrm{~g} \text { due to starch gelatinization for cookies; } \\
\text { Placebo: } 20 \mathrm{~g} \text { manioc flour daily. }\end{array}$ & 1 sachet daily & 4 weeks \\
\hline Khosroshahi 2018 [20] & Iran & $\begin{array}{l}22 \text { patients and } 22 \\
\text { controls }\end{array}$ & $\begin{array}{l}\text { RS2: } 20 \mathrm{~g} \text { or } 25 \mathrm{~g} \text { of } 60 \% \text { RS } 2 \text { in HAM-RS } 2 \text { enriched crackers; } \\
\text { Placebo: regular wheat flour. }\end{array}$ & 1 sachet daily & 8 weeks \\
\hline Khosroshahi 2019 [21] & Iran & $\begin{array}{l}23 \text { patients and } 21 \\
\text { controls }\end{array}$ & $\begin{array}{l}\text { RS2: } 20 \text { g or } 25 \mathrm{~g} \text { of } 60 \% \text { RS2 in HAM-RS2; } \\
\text { Placebo: } 20 \text { g or } 25 \mathrm{~g} \text { of waxy corn starch. }\end{array}$ & 1 sachet daily & 8 weeks \\
\hline Laffin 2019 [23] & Iran & $\begin{array}{l}9 \text { patients and } 11 \\
\text { controls }\end{array}$ & $\begin{array}{l}\text { RS2: } 20 \mathrm{~g} \text { or } 25 \mathrm{~g} \text { of } 60 \% \text { RS2 in HAM-RS } 2 \text { enriched crackers; } \\
\text { Placebo: regular wheat flour. }\end{array}$ & 1 sachet daily & 2 months \\
\hline
\end{tabular}

We extracted and analyzed the data of 58 patients treated with RS2 and 57 patients treated with placebo. SMD was used to summarize measures for parameters with different units among studies. No changes were found in levels of IS (SMD $=-0.33,95 \%$ CI: -0.70 to $0.04, I^{2}=0 \%, P=0.08$, Figure $4 a$ ) and PCS $\left(\mathrm{SMD}=-0.31,95 \% \mathrm{CI}:-0.68\right.$ to $0.06, I^{2}=0 \%, P=0.10$, Figure $4 b$ ) of the RS2 group compared with the placebo group. Thus, RS2 could not decrease the levels of uremic toxins of patients under MHD.

\section{Secondary outcomes}

The levels of IL-6 in 95 patients under MHD were measured in 3 studies. A significant decrease of IL-6 was found in the treatment group (SMD $=-1.08$, 95\% CI: -1.64 to $-0.53, I^{2}=35 \%, P=0.0001$, Figure $\left.5 a\right)$. However, the change of hsCRP was insignificant (SMD $=0.17,95 \%$ CI: -0.22 to $0.56, I^{2}=14 \%, P=0.40$, Figure $5 \mathbf{b}$ ). Malnutrition and hyperphosphatemia are two major complications of MHD [31]. Serum levels of albumin reflect the nutrition status of patients. We found insignificant changes of albumin in the RS2 group (WMD $=0.06,95 \%$ CI: -0.06 to $0.18, I^{2}=0 \%, P=$ 0.33 , Figure 6a). Neither was the level of phosphorus changed by the RS2 treatment (WMD $=-0.03,95 \% \mathrm{CI}$ : -0.36 to $0.30, I^{2}=0 \%, P=0.84$, Figure $6 \mathbf{b}$ ). 
starch type 2; Scr: serum creatinine; SD: standard deviation; UA: uric acid.

GRADE Working Group grades of evidence:

High quality: Further research is very unlikely to change our confidence in the estimate of effect;

Moderate quality: Further research is likely to have an important impact on our confidence in the estimate of effect and may change the estimate;

Low quality: Further research is very likely to have an important impact on our confidence in the estimate of effect and is likely to change the estimate;

Very low quality: Very uncertain about the estimate.

${ }^{1}$ Imprecise due to the small sample size (less than 300 ) in all studies. Thus, the evidence quality was down-graded as one level.

2 One study was inconsistent with the other three in BUN, thus the quality of evidence was down-graded by one level.

3 One study was supported by food company of RS2, thus the quality of evidence was down-graded by one level.

${ }^{4}$ One study was inconsistent with the other two in Scr, thus the quality of evidence was down-graded by one level.

${ }^{5}$ One study was inconsistent with the other two in UA, thus the quality of evidence was down-graded by one level.

${ }^{6}$ One study was inconsistent with the other two in IS, thus the quality of evidence was down-graded by one level.

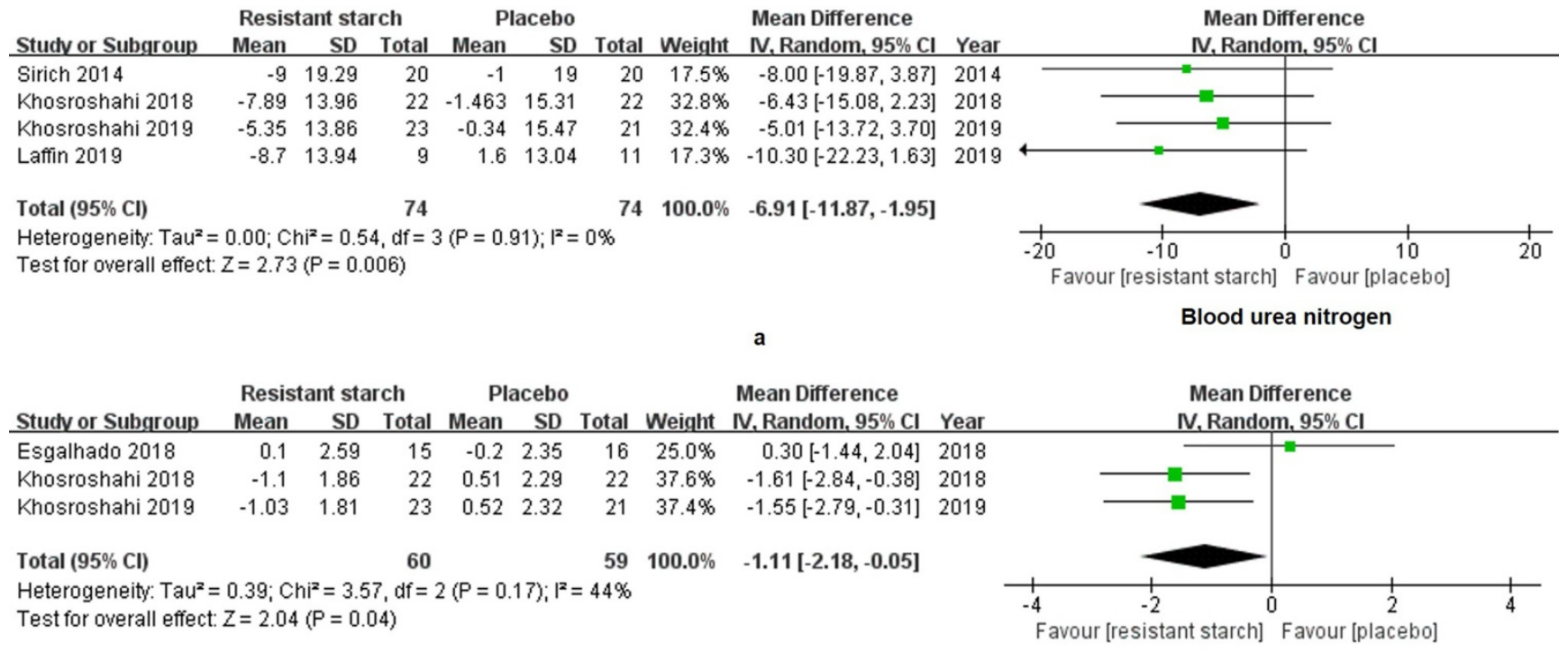

\begin{tabular}{|c|c|c|c|c|c|c|c|c|c|c|}
\hline \multirow[b]{3}{*}{ Studv or Subgroup } & \multicolumn{8}{|c|}{ b } & \multicolumn{2}{|l|}{ Serum creatine } \\
\hline & \multicolumn{3}{|c|}{ Resistant starch } & \multicolumn{3}{|c|}{ Placebo } & \multirow[b]{2}{*}{ Weight } & \multirow{2}{*}{$\begin{array}{l}\text { Mean Difference } \\
\text { IV, Random, } 95 \% \mathrm{Cl}\end{array}$} & \multirow{2}{*}{\multicolumn{2}{|c|}{$\begin{array}{c}\text { Mean Difference } \\
\text { IV, Random, 95\% Cl }\end{array}$}} \\
\hline & Mean & SD & Total & Mean & SD & Total & & & & \\
\hline Khosroshahi 2018 & -0.51 & 1.05 & 22 & -0.7 & 1.05 & 22 & $43.1 \%$ & $0.19[-0.43,0.81]$ & & \\
\hline Khosroshahi 2019 & -0.58 & 1.1 & 23 & -0.69 & 1.1 & 21 & $39.2 \%$ & $0.11[-0.54,0.76]$ & t마 & \\
\hline Laffin 2019 & -0.27 & 1.11 & 9 & -0.55 & 1.08 & 11 & $17.8 \%$ & $0.28[-0.69,1.25]$ & & \\
\hline Total $(95 \% \mathrm{Cl})$ & & & 54 & & & 54 & $100.0 \%$ & $0.17[-0.23,0.58]$ & & \\
\hline \multicolumn{7}{|c|}{$\begin{array}{l}\text { Heterogeneity: } \operatorname{Tau}^{2}=0.00 ; \mathrm{Chi}^{2}=0.09, \mathrm{df}=2(P=0.96) ; I^{2}=0 \% \\
\text { Test for overall effect: } Z=0.84(P=0.40)\end{array}$} & & & $\begin{array}{c}1 \\
-2\end{array}$ & 1 \\
\hline
\end{tabular}

Uric acid

Figure 3. Forest plots for comparisons of blood urea nitrogen (BUN), serum creatinine (Scr) and uric acid (UA). Data of BUN (a) in four studies including 148 subjects were analyzed. A significant decrease of BUN was shown in the resistant starch type 2 (RS2) group compared with the control group (WMD $=-6.91,95 \% \mathrm{Cl},-11.87$ to $\left.-1.95,1^{2}=0 \%, P=0.006\right)$. Scr $(\mathbf{b})$ also showed a decrease in the RS2 group compared with the placebo in the meta-analysis $(\mathrm{WMD}=-7.43,95 \% \mathrm{Cl},-11.99$ to $-2.86,12=44 \%, P$ $=0.001)$. However, no significant change of UA (c) has been displayed after RS2 treatment (WMD $=0.17,95 \% \mathrm{Cl},-0.23$ to $0.58,12=0 \%, P=0.40$ )

\section{Discussion}

Research has rarely been focused on the effectiveness of RS2 in patients under MHD. In the current study, we summarized clinical trials and investigated the therapeutic effects of RS2 on patients under MHD, including changes of Scr, BUN, UA, PCS, IS, IL-6, hs-CRP, albumin and phosphorus. We found that RS2 could improve the residual kidney function of patients under MHD by decreasing levels of Scr in very-low certainty and reducing BUN and IL-6 in low certainty. We also found that the levels of PCS, IS, hs-CRP, albumin and phosphorus were not significantly altered by the treatment with RS2.

Patients under MHD are frequently complicated with chronic inflammation, which has a great impact on the early morbidity and mortality of patients [32]. Chronic inflammation, together with medications, dietary changes and so forth, may result in alterations of the gut flora $[33,34]$. Gut dysbiosis results in an increased concentration of uremic toxins [35], leading to the inhibition of colonocyte proliferation, and even increasing DNA damage towards colonocytes $[36,37]$. The damaged gut barrier allows leakage of endotoxins into the circulation system [36], which facilitates the development of CKD by activating 
inflammatory cytokine production and aggravates chronic inflammation systemically and in kidney [38, 39]. Presumably, RS2 may act as a prebiotic to modulate the proportion of gut microbiota by increasing the ratio of beneficial bacteria, such as Lachnospiraceae, Ruminococcaeae, Faecalibacterium, etc $[23,40]$. This may further reduce the production of uremic toxins so as to improve the integrity of the intestinal barrier and to decrease the microinflammation in other organs [41]. Our meta-analysis showed that RS2 did benefit patients under MHD in terms of improving the residual kidney function; the reduced levels of Scr and BUN after RS2 treatment suggests that RS2 may prolong the interdialysis period. Younes et al previously reported that plasma urea concentrations were decreased both in normal and nephrectomized rats fed with fermentable carbohydrates, namely RS [42]. Later, researchers confirmed the function of RS2 in reducing BUN, Scr and other CKD-related biomarkers in other animal experiments [40,43]. Our findings are consistent with previous bench studies [40,43] and add knowledge to the benefit of RS2 for patients under MHD.

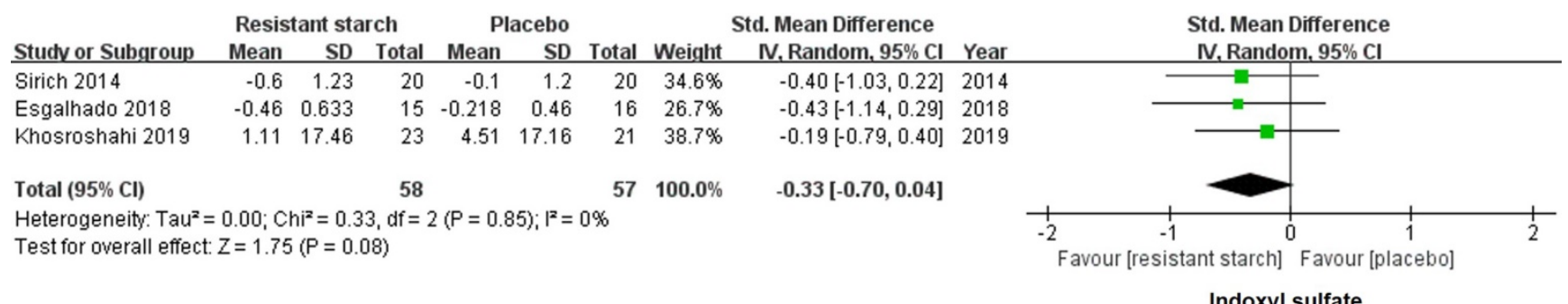

a

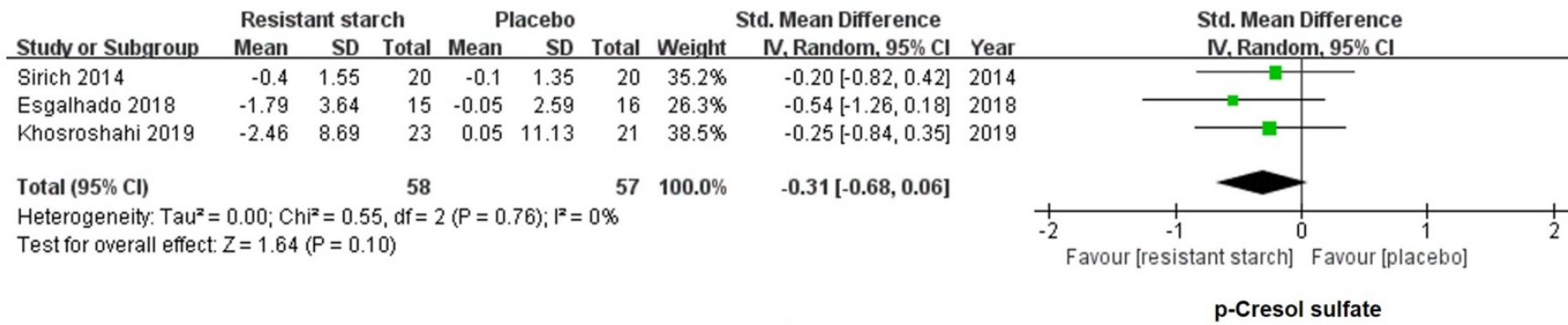

Figure 4. Forest plots for comparisons of indoxyl sulfate (IS) and p-cresyl sulfate (PCS) in serum and plasma. Sirich et al.'s, Esgalhado et al.'s and Khosroshahi 2019 et al.'s studies reported data of serum and plasma IS (a) and PCS (b) in resistant starch type 2 (RS2) and control patients under hemodialysis. Neither IS (SMD = -0.33, $95 \%$ $\mathrm{Cl},-0.70$ to $\left.0.04,1^{2}=0 \%, P=0.08\right)$ nor PCS (SMD $=-0.31,95 \% \mathrm{Cl},-0.68$ to $0.06,12=0 \%, P=0.10$ ) was changed significantly after treatment with $\mathrm{RS} 2$.

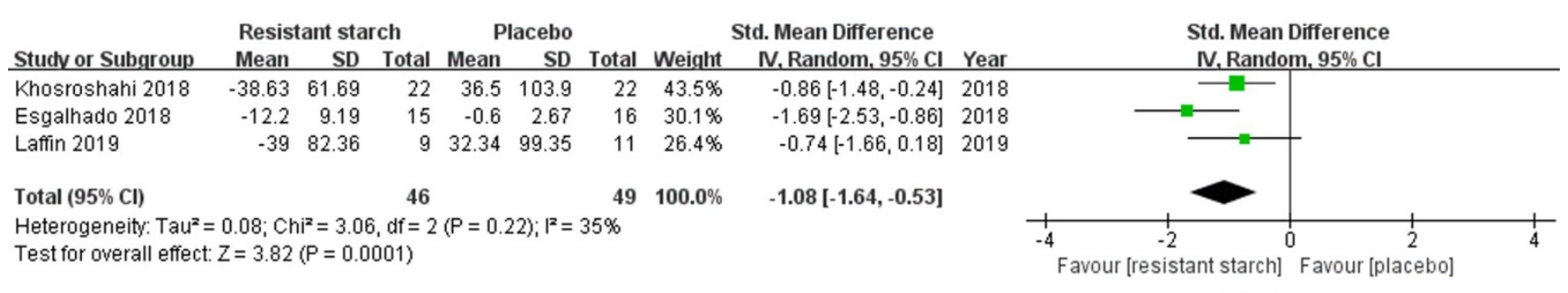

a

Interleukin 6

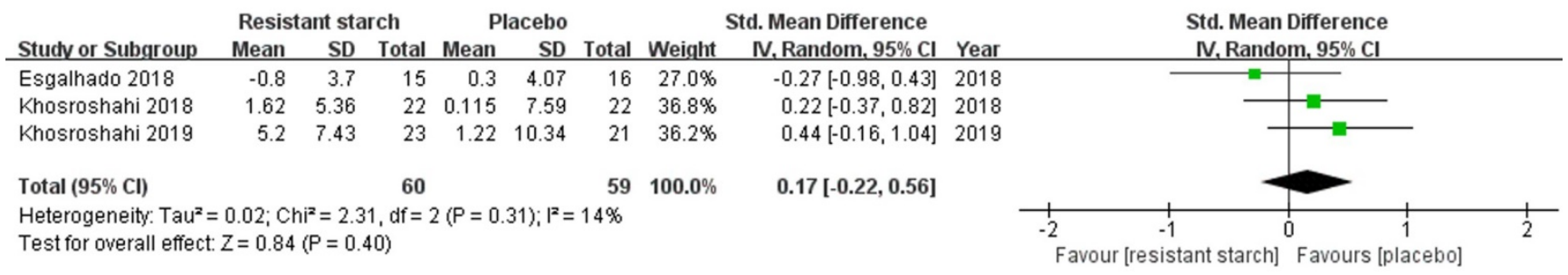

High sensitive $\mathrm{C}$ reactive protein

Figure 5. Forest plots for comparisons of interleukin (IL)-6 and high sensitive C-reaction protein (hsCRP) in serum. Three studies reported data of IL-6 in a total of 95 patients under maintenance hemodialysis (MHD). Resistant starch type 2 (RS2) could decrease the level of IL-6 in patients under MHD (SMD = -1.08, $95 \%$ CI: -1.64 to -0.53 , $12=35 \%, P=0.0001$, a). However, no significant change was found in serum level of hsCRP (SMD $=0.17,95 \% \mathrm{Cl}:-0.22$ to $0.56,12=14 \%, P=0.40, \mathbf{b})$. 


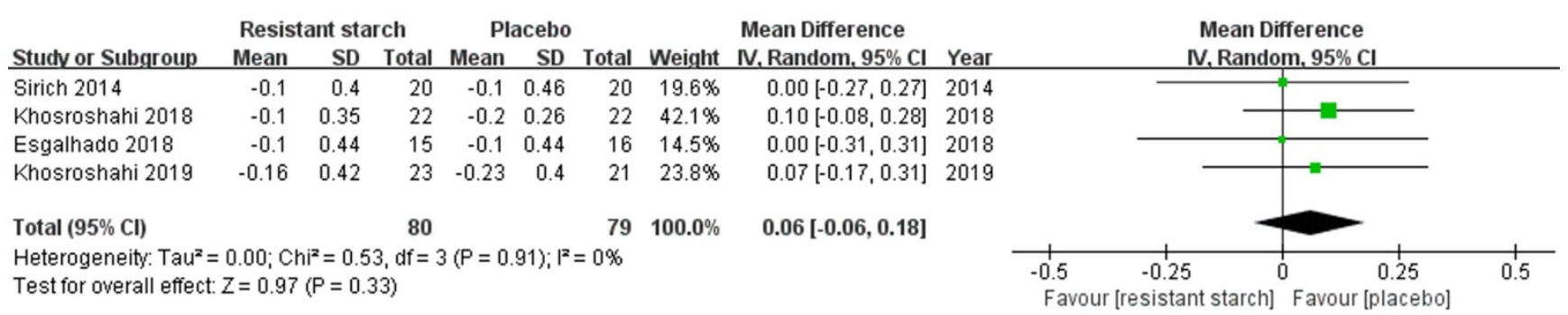

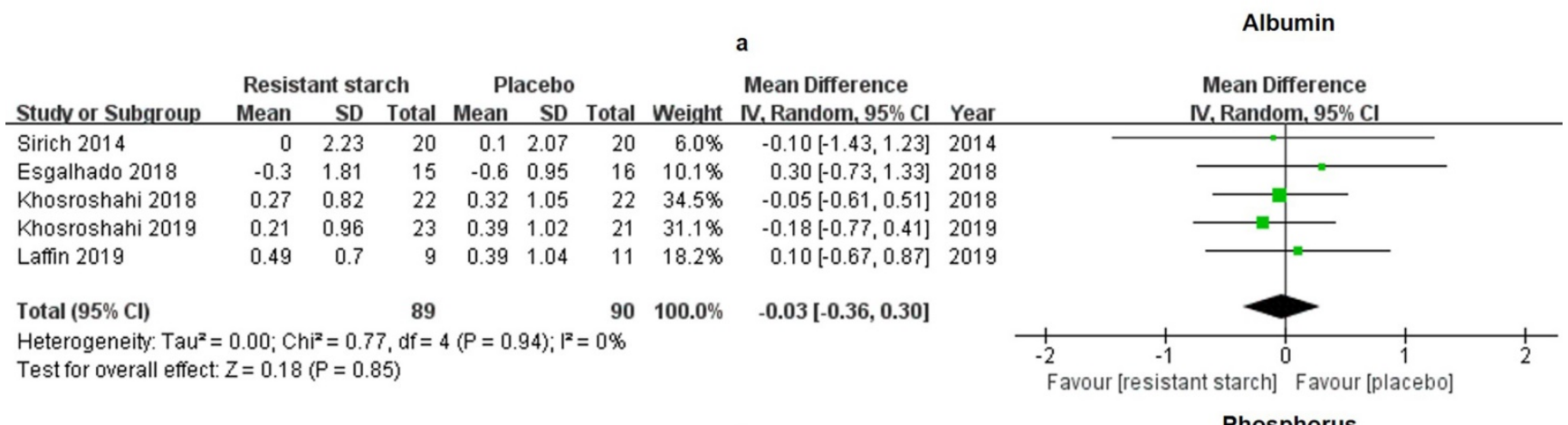

Figure 6. Forest plots for comparisons of albumin and phosphorus in serum. Four studies including 159 subjects reported the data of albumin (a). Levels of albumin were insignificantly altered in the resistant starch 2 group as compared with the control group (WMD $=0.06,95 \% \mathrm{Cl},-0.06$ to $-0.18,12=0 \%, P=0.33)$. As for phosphorus $(\mathbf{b})$, meta-analysis for five studies of 179 subjects showed insignificant change as well (WMD $=-0.03,95 \% \mathrm{Cl},-0.36$ to $0.30,12=0 \%, P=0.84)$.

PCS and IS are two major uremic toxins, except for Src and BUN, excreted by the kidney, which are metabolites of bacterial products of p-cresol and indole respectively [44]. PCS and IS are bound to plasma protein [45] and hence cannot be effectively eliminated by severely diseased kidneys or MHD [45]. PCS and IS may accumulate in patients with CKD and further aggravate micro-inflammation in the kidney $[46,47]$. Several animal studies demonstrate that RS2 adjusted the proportion of intestinal symbiotic microbiota and increased the production of SCFAs, which could decrease the levels of PCS and IS [43]. The effects of RS2 on PCS and IS were later corroborated by clinical trials [21, 22, 48]. Wu et al demonstrated that dietary fiber intake could reduce the level of PCS in 203 CKD patients via a meta-analysis [48]. Khosroshahi et al also revealed a significant decrease of PCS after RS2 treatment in patients under MHD [21]. Instead, Esgalhado et al reported a totally opposite finding [22]. In our metaanalysis of PCS and IS, neither of the two indicators was changed after treatment with RS2. Our results suggest that reduced levels of PCS and IS may not be the major mechanism underlying the suppressed inflammatory response after the treatment with RS2. Other factors or pathways may be implicated.

RS2 has been verified to reduce inflammatory biomarkers in various diseases [22]. In the present study, a significant decrease of IL-6 was noted in patients under MHD upon treatment with RS2. Another meta-analysis also indicated that levels of inflammatory responses could be reduced by RS2 in patients with metabolic syndrome [49]. Supplementation with RS2 to nephrectomized rats reduced nuclear factor kappa-B as well as proinflammatory molecules [50]. We hereby postulate IL-6 as the key factor in the therapeutic effects of RS2. IL-6 is an important cytokine in the progression of CKD. In previous studies, increased levels of IL-6 were found in renal tissues of CKD patients and were related to risk of CKD complications [51,52]. Honda et al demonstrated that IL-6 was a reliable predictor of CKD related malnutrition and cardiovascular diseases [53]. Kaminska et al suggested that the serum level of IL-6 was associated with coronary artery calcification and 5-year risk of all-cause mortality in CKD patients $[54,55]$. Importantly, renal cells, including podocytes, mesangial cells, endothelial cells and so forth, may express and secrete IL-6 [51]. In kidney, IL-6 could act as both a proinflammatory and an anti-inflammatory factor to promote cell proliferation, differentiation and tubulointerstitial fibrosis [56]. In CKD, IL-6 is mainly increased in response to kidney injury to promote an inflammatory response [57]. In specific, IL-6 activates signal transducer and activator of transcription 3 and increases the expression of fibroblast growth factor 23 in CKD [58], which further promotes the systemic inflammation and leads to the renal fibrosis $[59,60]$. In this regard, lowering the level of IL-6 in patients under MHD may alleviate disease process of ESRD. Since similar studies are limited, the detailed mechanism of IL-6 in RS2-treated patients under MHD merits further verification. 
Hyperphosphatemia frequently complicates patients under MHD [61]. Aggravated phosphorus burden in patients under MHD might be attributable to gut dysbiosis and consequent impaired activity of intracellular pathway of nicotinamide phosphoribosyltransferase and phosphate transporters in the intestines [61, 62]. RS2 was shown to reduce the levels of phosphorus in animal studies [63]. Indeed, RS2 might not directly participate in the metabolic pathway of phosphorus. The negative finding in the present study might be due to different levels of phosphorus intake, as the dietary recipe was not recorded in all of the included studies. This assumption may as well apply to albumin, an indicator of malnutrition; RS2 could neither increase nor reduce its serum levels.

When interpreting the results of this review, some limitations should be taken into account. First, the small sample size of the included studies may increase the reporting bias and undermine the evidence level. Second, because of insufficient data, effects of RS2 treatment on clinical endpoint events, death for instance, cardiovascular disease events and so forth, cannot be deciphered. Third, difference in duration and dosage of RS2 supplement may influence the efficacy in the meta-analysis; these discrepancies make it difficult to attain strong evidence for guiding clinical practice. Finally, all data were extracted from published articles. We did not contact authors for original data, which may render our meta-analysis subject to publication bias.

In conclusion, our results suggest that dietary supplement with RS2 may improve the residual renal function of patients under MHD by reducing inflammatory mediator IL-6. Nevertheless, results should be cautiously interpreted, because of the limited sample size and different treatment dosages. Large and pragmatic multicenter trials are thus necessary to corroborate the beneficial effects of RS2 supplementation on ESRD.

\section{Acknowledgements}

\section{Funding sources}

The study was supported by grants from National Natural Science Foundation of China (No. 81900655), Wu Jieping Medical Foundation (No. 320.6750.16050), Beijing Excellent Talents Foundation (No. 2018000020124G144), and Scientific Research Project of Beijing Educational Committee (No. KM202010025022). Funders play no role in this systematic review.

\section{Statement of authorship}

LJ, RJ and HLZ designed the study. XD and LJ designed the search strategy and searched the publications. XL, HLZ and RJ assessed the studies. XL, HLZ and RJ extracted the data. LJ, XD and HLZ assessed the risk of bias and the quality of evidence. LJ and HLZ analyzed and interpreted the data. LJ and RJ drafted the article. HLZ critically revised the manuscript. All authors approved the final version for submission and publication. Zhang HL is affiliated with the National Natural Science Foundation of China. The views expressed are his own and do not necessarily represent the views of the National Natural Science Foundation of China or the Chinese government.

\section{Competing Interests}

The authors have declared that no competing interest exists.

\section{References}

1. Webster AC, Nagler EV, Morton RL, et al. Chronic Kidney Disease. Lancet. 2017;389(10075):1238-52.

2. Zhang L, Wang F, Wang L, et al. Prevalence of chronic kidney disease in China: a cross-sectional survey. Lancet. 2012;379(9818):815-22.

3. Hole B, Tonkin-Crine S, Caskey FJ, et al. Treatment of End-stage Kidney Failure without Renal Replacement Therapy. Semin Dial. 2016;29(6):491-506.

4. Wolfgram DF. Intradialytic Cerebral Hypoperfusion as Mechanism for Cognitive Impairment in Patients on Hemodialysis. J Am Soc Nephrol. 2019;30(11):2052-8.

5. Furman D, Campisi J, Verdin E, et al. Chronic inflammation in the etiology of disease across the life span. Nat Med. 2019;25(12):1822-32.

6. Panichi V, Rizza GM, Paoletti S, et al. Chronic inflammation and mortality in haemodialysis: effect of different renal replacement therapies. Results from the RISCAVID study. Nephrol Dial Transplant. 2008;23(7):2337-43.

7. Zhou $\mathrm{Y}, \mathrm{Xu} \mathrm{H}$, Huang $\mathrm{H}$, et al. Are There Potential Applications of Fecal Microbiota Transplantation beyond Intestinal Disorders? Biomed Res Int. 2019:2019:3469754.

8. Vaziri ND, Wong J, Pahl M, et al. Chronic kidney disease alters intestinal microbial flora. Kidney Int. 2013;83(2):308-15.

9. Ramezani A, Raj DS. The gut microbiome, kidney disease, and targeted interventions. J Am Soc Nephrol. 2014;25(4):657-70.

10. Mihai S, Codrici E, Popescu ID, et al. Inflammation-Related Mechanisms in Chronic Kidney Disease Prediction, Progression, and Outcome. J Immunol Res. 2018;2018:2180373.

11. de Almeida Duarte JB, de Aguilar-Nascimento JE, Nascimento M, et al. Bacterial translocation in experimental uremia. Urol Res. 2004;32(4):266-70.

12. Noce A, Marrone G, Di Daniele F, et al. Impact of Gut Microbiota Composition on Onset and Progression of Chronic Non-Communicable Diseases. Nutrients. 2019;11(5):1073.

13. Bindels LB, Segura Munoz RR, Gomes-Neto JC, et al. Resistant starch can improve insulin sensitivity independently of the gut microbiota. Microbiome. 2017;5(1):12

14. Harris KF. An introductory review of resistant starch type 2 from highamylose cereal grains and its effect on glucose and insulin homeostasis. Nutr Rev. 2019:pii: nuz040.

15. Yang X, Darko KO, Huang Y, et al. Resistant Starch Regulates Gut Microbiota: Structure, Biochemistry and Cell Signalling. Cell Physiol Biochem. 2017;42(1):306-18

16. Cerqueira FM, Photenhauer AL, Pollet RM, et al. Starch Digestion by Gut Bacteria: Crowdsourcing for Carbs. Trends Microbiol. 2020;28(2):95-108.

17. Moraes $C$, Borges NA Mafra D. Resistant starch for modulation of gut microbiota: Promising adjuvant therapy for chronic kidney disease patients? Eur J Nutr. 2016;55(5):1813-21.

18. Khoueiry G, Waked A, Goldman M, et al. Dietary intake in hemodialysis patients does not reflect a heart healthy diet. J Ren Nutr. 2011;21(6):438-47.

19. Sirich TL, Plummer NS, Gardner CD, et al. Effect of increasing dietary fiber on plasma levels of colon-derived solutes in hemodialysis patients. Clin J Am Soc Nephrol. 2014.9(9):1603-10.

20. Tayebi Khosroshahi H, Vaziri ND, Abedi B, et al. Effect of high amylose resistant starch (HAM-RS2) supplementation on biomarkers of inflammation and oxidative stress in hemodialysis patients: a randomized clinical trial. Hemodial Int. 2018;22(4):492-500

21. Khosroshahi HT, Abedi B, Ghojazadeh M, et al. Effects of fermentable high fiber diet supplementation on gut derived and conventional nitrogenous product in patients on maintenance hemodialysis: a randomized controlled trial. Nutr Metab (Lond). 2019;16:18 
22. Esgalhado M, Kemp JA, Azevedo $R$, et al. Could resistant starch supplementation improve inflammatory and oxidative stress biomarkers and uremic toxins levels in hemodialysis patients? A pilot randomized controlled trial. Food Funct. 2018;9(12):6508-16

23. Laffin MR, Tayebi Khosroshahi H, Park H, et al. Amylose resistant starch (HAM-RS2) supplementation increases the proportion of Faecalibacterium bacteria in end-stage renal disease patients: Microbial analysis from a randomized placebo-controlled trial. Hemodial Int. 2019;23(3):343-7.

24. Johansen KL, Dalrymple LS, Delgado C, et al. Comparison of self-report-based and physical performance-based frailty definitions among patients receiving maintenance hemodialysis. Am J Kidney Dis. 2014;64(4):600-7.

25. Higgins JP, Altman DG, Gotzsche PC, et al. The Cochrane Collaboration's tool for assessing risk of bias in randomised trials. BMJ. 2011;343:d5928.

26. Holger J Schünemann, Andrew D Oxman, Gunn E Vist, et al. Interpreting results and drawing conclusions. In: Julian PT Higgins, Green S, editors. Cochrane Handbook for Systematic Reviews of Intervention. 2019; https://doi.org/10.1002/9781119536604.ch15

27. Saglimbene VM, Wong G, van Zwieten A, et al. Effects of omega-3 polyunsaturated fatty acid intake in patients with chronic kidney disease: Systematic review and meta-analysis of randomized controlled trials. Clin Nutr. 2020;39(2):358-68.

28. Higgins JP, Thompson SG, Deeks JJ, et al. Measuring inconsistency in metaanalyses. BMJ. 2003;327(7414):557-60.

29. Snelson M, Kellow NJ, Coughlan MT. Modulation of the Gut Microbiota by Resistant Starch as a Treatment of Chronic Kidney Diseases: Evidence of Efficacy and Mechanistic Insights. Adv Nutr. 2019;10(2):303-20.

30. Kieffer DA, Martin RJ, Adams SH. Impact of Dietary Fibers on Nutrient Management and Detoxification Organs: Gut, Liver, and Kidneys. Adv Nutr. 2016;7(6):1111-21.

31. Salhab N, Alrukhaimi M, Kooman J, et al. Effect of Intradialytic Exercise on Hyperphosphatemia and Malnutrition. Nutrients. 2019;11(10):2464.

32. Morinaga J, Kakuma T, Fukami H, et al. Circulating angiopoietin-like protein 2 levels and mortality risk in patients receiving maintenance hemodialysis: a prospective cohort study. Nephrol Dial Transplant. 2019.

33. Lun $\mathrm{H}$, Yang $\mathrm{W}$, Zhao $\mathrm{S}$, et al. Altered gut microbiota and microbial biomarkers associated with chronic kidney disease. Microbiologyopen. 2019;8(4):e00678.

34. Simoes-Silva L, Araujo R, Pestana M, et al. The microbiome in chronic kidney disease patients undergoing hemodialysis and peritoneal dialysis. Pharmacol Res. 2018;130:143-51.

35. Kanbay M, Onal EM, Afsar B, et al. The crosstalk of gut microbiota and chronic kidney disease: role of inflammation, proteinuria, hypertension, and diabetes mellitus. Int Urol Nephrol. 2018;50(8):1453-66.

36. Ramezani A, Massy ZA, Meijers B, et al. Role of the Gut Microbiome in Uremia: A Potential Therapeutic Target. Am J Kidney Dis. 2016;67(3):483-98.

37. Andriamihaja M, Lan A, Beaumont $\mathrm{M}$, et al. The deleterious metabolic and genotoxic effects of the bacterial metabolite p-cresol on colonic epithelial cells. Free Radic Biol Med. 2015;85:219-27.

38. Freudenberg MA, Tchaptchet S, Keck S, et al. Lipopolysaccharide sensing an important factor in the innate immune response to Gram-negative bacterial infections: benefits and hazards of LPS hypersensitivity. Immunobiology. 2008;213(3-4):193-203.

39. Koeth RA, Wang Z, Levison BS, et al. Intestinal microbiota metabolism of L-carnitine, a nutrient in red meat, promotes atherosclerosis. Nat Med. 2013;19(5):576-85.

40. Zybailov BL, Glazko GV, Rahmatallah Y, et al. Metaproteomics reveals potential mechanisms by which dietary resistant starch supplementation attenuates chronic kidney disease progression in rats. PLoS ONE. 2019;14(1):e0199274.

41. Yang T, Richards EM, Pepine CJ, et al. The gut microbiota and the brain-gut-kidney axis in hypertension and chronic kidney disease. Nat Rev Nephrol. 2018;14(7):442-56.

42. Younes H, Remesy C, Behr S, et al. Fermentable carbohydrate exerts a urealowering effect in normal and nephrectomized rats. Am J Physiol. 1997;272(3):G515-G21.

43. Kieffer DA, Piccolo BD, Vaziri ND, et al. Resistant starch alters gut microbiome and metabolomic profiles concurrent with amelioration of chronic kidney disease in rats. Am J Physiol Renal Physiol. 2016;310(9):F857-71.

44. Cosola C, De Angelis M, Rocchetti MT, et al. Beta-Glucans supplementation associates with reduction in P-cresyl sulfate levels and improved Endothelial Vascular Reactivity in Healthy Individuals. PLoS One. 2017;12(1):e0169635.

45. Lesaffer $G$, De Smet $R$, Lameire $N$, et al. Intradialytic removal of proteinbound uraemic toxins: role of solute characteristics and of dialyser membrane. Nephrol Dial Transplant. 2000;15(1):50-7.

46. Jia L, Jia Q, Yang J, et al. Efficacy of Probiotics Supplementation On Chronic Kidney Disease: a Systematic Review and Meta-Analysis. Kidney Blood Press Res. 2018;43(5):1623-35

47. Ito S, Yoshida M. Protein-bound uremic toxins: new culprits of cardiovascular events in chronic kidney disease patients. Toxins (Basel). 2014;6(2):665-78.

48. Wu M, Cai X, Lin J, et al. Association between fibre intake and indoxyl sulphate/P-cresyl sulphate in patients with chronic kidney disease: Metaanalysis and systematic review of experimental studies. Clin Nutr. 2019;38(5):2016-22

49. Halajzadeh J, Milajerdi A, Reiner Z, et al. Effects of resistant starch on glycemic control, serum lipoproteins and systemic inflammation in patients with metabolic syndrome and related disorders: A systematic review and meta-analysis of randomized controlled clinical trials. Crit Rev Food Sci Nutr. 2019;p:1-13.

50. Vaziri ND, Liu SM, Lau WL, et al. High amylose resistant starch diet ameliorates oxidative stress, inflammation, and progression of chronic kidney disease. PLoS One. 2014;9(12):e114881.

51. Magno AL, Herat LY, Carnagarin R, et al. Current Knowledge of IL-6 Cytokine Family Members in Acute and Chronic Kidney Disease. Biomedicines. 2019;7(1):E19.

52. Turon-Skrzypinska A, Dutkiewicz G, Marchelek-Mysliwiec M, et al. Assessment of Sclerostin and Interleukin 6 Levels and Selected Anthropometric Parameters in Patients Receiving Hemodialysis Replacement Therapy-Pilot Study. Medicina (Kaunas). 2019;55(12):E784.

53. Honda $\mathrm{H}$, Qureshi $A R$, Heimburger $\mathrm{O}$, et al. Serum albumin, C-reactive protein, interleukin 6, and fetuin a as predictors of malnutrition, cardiovascular disease, and mortality in patients with ESRD. Am J Kidney Dis. 2006;47(1):139-48.

54. Kaminska J, Stopinski M, Mucha $\mathrm{K}$, et al. IL 6 but not TNF is linked to coronary artery calcification in patients with chronic kidney disease. Cytokine. 2019;120:9-14.

55. Munoz Mendoza J, Isakova T, Cai X, et al. Inflammation and elevated levels of fibroblast growth factor 23 are independent risk factors for death in chronic kidney disease. Kidney Int. 2017;91(3):711-9.

56. Su H, Lei CT, Zhang C. Interleukin-6 Signaling Pathway and Its Role in Kidney Disease: An Update. Front Immunol. 2017;8:405

57. Nechemia-Arbely Y, Barkan D, Pizov G, et al. IL-6/IL-6R axis plays a critical role in acute kidney injury. J Am Soc Nephrol. 2008;19(6):1106-15.

58. Durlacher-Betzer $K$, Hassan A, Levi $R$, et al. Interleukin-6 contributes to the increase in fibroblast growth factor 23 expression in acute and chronic kidney disease. Kidney Int. 2018;94(2):315-25.

59. Zhu $\mathrm{Q}$, Zeng DK, Li FQ. FGF23 promotes renal interstitial fibrosis by activating beta-catenin. Eur Rev Med Pharmacol Sci. 2018;22(1):174-83.

60. Chen W, Yuan H, Cao W, et al. Blocking interleukin-6 trans-signaling protects against renal fibrosis by suppressing STAT3 activation. Theranostics. 2019:9(14):3980-91.

61. Barreto FC, Barreto DV, Massy ZA, et al. Strategies for Phosphate Control in Patients With CKD. Kidney Int Rep. 2019;4(8):1043-56.

62. Rahbar Saadat Y, Niknafs B, Hosseiniyan Khatibi SM, et al. Gut microbiota; an overlooked effect of phosphate binders. Eur J Pharmacol. 2020;868:172892.

63. Rideout TC, Liu Q, Wood $P$, et al. Nutrient utilisation and intestinal fermentation are differentially affected by the consumption of resistant starch varieties and conventional fibres in pigs. Br J Nutr. 2008;99(5):984-92. 